\title{
Time-Domain Calculations of the Infrared and Polarized Raman Spectra of Tetraalanine in Aqueous Solution
}

\author{
Hajime Torii * \\ Department of Chemistry, School of Education, Shizuoka University, \\ 836 Ohya, Shizuoka 422-8529, Japan \\ * Telephone and Fax: +81-54-238-4624; E-mail: torii@ed.shizuoka.ac.jp.
}

\section{Supporting Information \\ Derivation of the limiting value of $M(t)$ upon complete mode mixing of a three-mode system}

The limiting value of $M(t)$ (defined by eq 10 in section 3B) upon complete mode mixing of a three-mode system, denoted as $M_{\mathrm{cmm}}^{(3)}$, is calculated as the average of $\cos ^{2} \theta$ in the region where $\cos ^{2} \theta$ is larger than $\sin ^{2} \theta \cos ^{2} \psi$ and $\sin ^{2} \theta \sin ^{2} \psi$, where $\theta$ and $\psi$ (as well as $\varphi$ ) are the Euler angles of three-dimensional coordinate transformation. This region is denoted as $\Gamma$ hereafter. Therefore, $M_{\mathrm{cmm}}^{(3)}$ is expressed as

$$
M_{\mathrm{cmm}}^{(3)}=\int_{\Gamma} d \Omega \cos ^{2} \theta / \int_{\Gamma} d \Omega
$$

Without losing generality and preciseness, we restrict our calculation to the upper hemisphere of $\theta(0 \leq \theta \leq \pi / 2)$, and omit the integration over $\varphi$.

$\Gamma$ is divided into two sub-regions: $\Gamma_{1}$, where $\cos ^{2} \theta$ is larger than $\sin ^{2} \theta(0 \leq \theta \leq \pi / 4)$, and $\Gamma_{2}$, where $\cos ^{2} \theta$ is smaller than $\sin ^{2} \theta$ but larger than $(1 / 2) \sin ^{2} \theta[\pi / 4 \leq \theta \leq$ $\left.\cos ^{-1}(1 / \sqrt{3})\right]$ with a condition also for $\psi$. The integration in $\Gamma_{1}$ is rather straightforward. At any value of $\theta$ in the range of $\pi / 4 \leq \theta \leq \cos ^{-1}(1 / \sqrt{3})$, the width of the range of $\psi$ 
satisfying the condition that $\cos ^{2} \theta$ is larger than $\sin ^{2} \theta \cos ^{2} \psi$ and $\sin ^{2} \theta \sin ^{2} \psi$ is $2 \pi-$ $8 \cos ^{-1}(\cot \theta)$. Therefore, the denominator of eq $\mathrm{S} 1$ is expressed as

$$
\int_{\Gamma} d \Omega=2 \pi\left(1-\frac{1}{\sqrt{3}}\right)-8 F\left[\pi / 4, \cos ^{-1}(1 / \sqrt{3}), 0\right]
$$

where $F[a, b, n]$ is defined as

$$
F[a, b, n] \equiv \int_{a}^{b} d \theta \sin \theta \cos ^{n} \theta \cos ^{-1}(\cot \theta)
$$

Changing the integration variable to $\xi=\cos ^{-1}(\cot \theta)$, we have

$$
F\left[\pi / 4, \cos ^{-1}(1 / \sqrt{3}), n\right]=\int_{0}^{\pi / 4} d \xi \frac{\sin \xi}{\left(1+\cos ^{2} \xi\right)^{3 / 2}}\left(\frac{\cos ^{2} \xi}{1+\cos ^{2} \xi}\right)^{n / 2} \xi
$$

In fact, as easily recognized, $\Gamma$ occupies one third of the hemisphere, so that eq S2 is equal to $2 \pi / 3$. We therefore obtain

$$
F\left[\pi / 4, \cos ^{-1}(1 / \sqrt{3}), 0\right]=\frac{\pi}{12}(2-\sqrt{3})
$$

The numerator of eq $\mathrm{S} 1$ is expressed as

$$
\int_{\Gamma} d \Omega \cos ^{2} \theta=\frac{2 \pi}{3}\left(1-\frac{1}{3 \sqrt{3}}\right)-8 F\left[\pi / 4, \cos ^{-1}(1 / \sqrt{3}), 2\right]
$$

Integrating eq $\mathrm{S} 4$ for $n=2$ by parts, we have

$$
\begin{aligned}
F\left[\pi / 4, \cos ^{-1}(1 / \sqrt{3}), 2\right]=\left[\frac{\cos \xi}{3\left(1+\cos ^{2} \xi\right)^{3 / 2}} \xi\right]_{0}^{\pi / 4} \\
+\frac{1}{3} \int_{0}^{\pi / 4} d \xi \frac{\sin \xi}{\left(1+\cos ^{2} \xi\right)^{3 / 2}} \xi-\frac{1}{3} \int_{0}^{\pi / 4} d \xi \frac{\cos \xi}{\left(1+\cos ^{2} \xi\right)^{3 / 2}}
\end{aligned}
$$

The first and second terms on the right-hand side are equal to $\sqrt{3} \pi / 54$ and $(1 / 3) F\left[\pi / 4, \cos ^{-1}(1 / \sqrt{3}), 0\right] \quad[=\pi(2-\sqrt{3}) / 36$ according to eq $\mathrm{S} 5]$, respectively. The integral in the third term is evaluated by changing the integration variable to $u=\sin \xi$ as 


$$
\int_{0}^{\pi / 4} d \xi \frac{\cos \xi}{\left(1+\cos ^{2} \xi\right)^{3 / 2}}=\int_{0}^{1 / \sqrt{2}} d u \frac{1}{\left(2-u^{2}\right)^{3 / 2}}=\frac{1}{2 \sqrt{3}}
$$

As a result, $M_{\mathrm{cmm}}^{(3)}$ is calculated as

$$
M_{\mathrm{cmm}}^{(3)}=\frac{1}{3}\left(1+\frac{2 \sqrt{3}}{\pi}\right) \cong 0.701
$$

